\title{
Synthesis, Structure and Upconversion Studies on Organically Templated Uranium Phosphites
}

\author{
Sukhendu Mandal ${ }^{\mathrm{a}}$, Manabendra Chandra ${ }^{\mathrm{b}}$, and Srinivasan Natarajan ${ }^{\mathrm{a}^{*}}$ \\ ${ }^{a}$ Framework Solids Laboratory, Solid State and Structural Chemistry Unit, Indian Institute of Science, \\ Bangalore 560012, India \\ and \\ ${ }^{\mathrm{b}}$ Department of Inorganic and Physical Chemistry, Indian Institute of Science, Bangalore 560012, India.
}

\section{Supplementary Materials}

\footnotetext{
${ }^{*}$ E-mail: snatarajan@,sscu.iisc.ernet.in
} 
Table 1. Selected bond distances in the compound $\left[\mathrm{C}_{4} \mathrm{~N}_{2} \mathrm{H}_{12}\right]\left[\left(\mathrm{U}^{\mathrm{VI}} \mathrm{O}_{2}\right)_{2} \mathrm{~F}_{2}\left(\mathrm{HPO}_{3}\right)_{2}\right], \mathbf{3}$.

\begin{tabular}{llll}
\hline Bond & Distance $(\AA)$ & Bond & Distance $(\AA)$ \\
\hline $\mathrm{U}(1)-\mathrm{O}(1)$ & $1.772(9)$ & $\mathrm{U}(1)-\mathrm{F}(1) \# 3$ & $2.388(6)$ \\
$\mathrm{U}(1)-\mathrm{O}(2)$ & $\mathrm{U}(1)-\mathrm{F}(1)$ & $2.405(6)$ \\
$\mathrm{U}(1)-\mathrm{O}(3)$ & $1.773(9)$ & $\mathrm{P}(1)-\mathrm{O}(3)$ & $1.515(9)$ \\
$\mathrm{U}(1)-\mathrm{O}(4) \# 1$ & $2.288(8)$ & $\mathrm{P}(1)-\mathrm{O}(4)$ & $1.515(9)$ \\
$\mathrm{U}(1)-\mathrm{O}(5) \# 2$ & $2.301(8)$ & $\mathrm{P}(1)-\mathrm{O}(5)$ & $1.549(8)$ \\
\hline
\end{tabular}

Symmetry transformations used to generate equivalent atoms: $\# 1-\mathrm{x}+1,-\mathrm{y}+1,-\mathrm{z}+1 ; \# 2 \mathrm{x}, \mathrm{y}-1, \mathrm{z} ; \# 3-\mathrm{x}+2,-$ $\mathrm{y}+1,-\mathrm{z}+2$ 
Table 2. Selected bond angles in $\left[\mathrm{C}_{2} \mathrm{~N}_{2} \mathrm{H}_{10}\right]\left[\mathrm{U}_{2}^{\mathrm{IV}} \mathrm{F}_{6}\left(\mathrm{HPO}_{3}\right)_{2}\right], \mathbf{1}, \quad\left[\mathrm{C}_{4} \mathrm{~N}_{2} \mathrm{H}_{12}\right]\left[\mathrm{U}_{2}{ }^{\mathrm{IV}} \mathrm{F}_{6}\left(\mathrm{HPO}_{3}\right)_{2}\right], \mathbf{2}$ and $\left[\mathrm{C}_{4} \mathrm{~N}_{2} \mathrm{H}_{12}\right]\left[\left(\mathrm{U}^{\mathrm{VI}} \mathrm{O}_{2}\right)_{2} \mathrm{~F}_{2}\left(\mathrm{HPO}_{3}\right)_{2}\right], \mathbf{3}$.

\begin{tabular}{|c|c|c|c|}
\hline Moiety & Angle ( ${ }^{\circ}$ ) & Moiety & Angle $\left({ }^{\circ}\right)$ \\
\hline \multicolumn{4}{|c|}{ Compound 1} \\
\hline $\mathrm{F}(1)-\mathrm{U}(1)-\mathrm{O}(1)$ & $78.9(2)$ & $\mathrm{F}(3) \# 1-\mathrm{U}(1)-\mathrm{F}(2) \# 2$ & $123.50(18)$ \\
\hline $\mathrm{F}(1)-\mathrm{U}(1)-\mathrm{O}(2)$ & $145.1(2)$ & $\mathrm{F}(1)-\mathrm{U}(1)-\mathrm{U}(1) \# 2$ & $73.35(16)$ \\
\hline $\mathrm{O}(1)-\mathrm{U}(1)-\mathrm{O}(2)$ & $86.4(3)$ & $\mathrm{O}(1)-\mathrm{U}(1)-\mathrm{U}(1) \# 2$ & $112.89(19)$ \\
\hline $\mathrm{F}(1)-\mathrm{U}(1)-\mathrm{F}(2)$ & $78.4(2)$ & $\mathrm{O}(2)-\mathrm{U}(1)-\mathrm{U}(1) \# 2$ & $83.76(16)$ \\
\hline $\mathrm{O}(1)-\mathrm{U}(1)-\mathrm{F}(2)$ & $144.9(2)$ & $\mathrm{F}(2)-\mathrm{U}(1)-\mathrm{U}(1) \# 2$ & $34.16(12)$ \\
\hline $\mathrm{O}(2)-\mathrm{U}(1)-\mathrm{F}(2)$ & $97.3(2)$ & $\mathrm{O}(3)-\mathrm{U}(1)-\mathrm{U}(1) \# 2$ & $118.2(2)$ \\
\hline $\mathrm{F}(1)-\mathrm{U}(1)-\mathrm{O}(3)$ & $71.4(2)$ & $\mathrm{F}(3)-\mathrm{U}(1)-\mathrm{U}(1) \# 2$ & $158.18(13)$ \\
\hline $\mathrm{O}(1)-\mathrm{U}(1)-\mathrm{O}(3)$ & $108.0(3)$ & $\mathrm{F}(3) \# 1-\mathrm{U}(1)-\mathrm{U}(1) \# 2$ & $99.94(12)$ \\
\hline $\mathrm{O}(2)-\mathrm{U}(1)-\mathrm{O}(3)$ & $143.5(2)$ & $\mathrm{F}(2) \# 2-\mathrm{U}(1)-\mathrm{U}(1) \# 2$ & $32.46(12)$ \\
\hline $\mathrm{F}(2)-\mathrm{U}(1)-\mathrm{O}(3)$ & $89.6(3)$ & $\mathrm{F}(1)-\mathrm{U}(1)-\mathrm{U}(1) \# 1$ & $141.74(15)$ \\
\hline $\mathrm{F}(1)-\mathrm{U}(1)-\mathrm{F}(3)$ & $128.5(2)$ & $\mathrm{O}(1)-\mathrm{U}(1)-\mathrm{U}(1) \# 1$ & $108.30(18)$ \\
\hline $\mathrm{O}(1)-\mathrm{U}(1)-\mathrm{F}(3)$ & $76.3(2)$ & $\mathrm{O}(2)-\mathrm{U}(1)-\mathrm{U}(1) \# 1$ & $72.98(15)$ \\
\hline $\mathrm{O}(2)-\mathrm{U}(1)-\mathrm{F}(3)$ & $77.0(2)$ & $\mathrm{F}(2)-\mathrm{U}(1)-\mathrm{U}(1) \# 1$ & $106.09(12)$ \\
\hline $\mathrm{F}(2)-\mathrm{U}(1)-\mathrm{F}(3)$ & $138.54(17)$ & $\mathrm{O}(3)-\mathrm{U}(1)-\mathrm{U}(1) \# 1$ & $70.66(17)$ \\
\hline $\mathrm{O}(3)-\mathrm{U}(1)-\mathrm{F}(3)$ & $74.4(2)$ & $\mathrm{F}(3)-\mathrm{U}(1)-\mathrm{U}(1) \# 1$ & $32.63(12)$ \\
\hline $\mathrm{F}(1)-\mathrm{U}(1)-\mathrm{F}(3) \# 1$ & $134.5(2)$ & $\mathrm{F}(3) \# 1-\mathrm{U}(1)-\mathrm{U}(1) \# 1$ & $32.61(12)$ \\
\hline $\mathrm{O}(1)-\mathrm{U}(1)-\mathrm{F}(3) \# 1$ & $139.9(2)$ & $\mathrm{F}(2) \# 2-\mathrm{U}(1)-\mathrm{U}(1) \# 1$ & $143.44(13)$ \\
\hline $\mathrm{O}(2)-\mathrm{U}(1)-\mathrm{F}(3) \# 1$ & $74.5(2)$ & $\mathrm{U}(1) \# 2-\mathrm{U}(1)-\mathrm{U}(1) \# 1$ & $130.80(2)$ \\
\hline $\mathrm{F}(2)-\mathrm{U}(1)-\mathrm{F}(3) \# 1$ & $73.60(17)$ & $\mathrm{O}(1) \# 3-\mathrm{P}(1)-\mathrm{O}(3) \# 1$ & $111.2(4)$ \\
\hline $\mathrm{O}(3)-\mathrm{U}(1)-\mathrm{F}(3) \# 1$ & $73.2(3)$ & $\mathrm{O}(1) \# 3-\mathrm{P}(1)-\mathrm{O}(2)$ & $111.4(4)$ \\
\hline $\mathrm{F}(3)-\mathrm{U}(1)-\mathrm{F}(3) \# 1$ & $65.2(2)$ & $\mathrm{O}(3) \# 1-\mathrm{P}(1)-\mathrm{O}(2)$ & $113.9(4)$ \\
\hline $\mathrm{F}(1)-\mathrm{U}(1)-\mathrm{F}(2) \# 2$ & $74.0(2)$ & $\mathrm{P}(1) \# 3-\mathrm{O}(1)-\mathrm{U}(1)$ & $147.2(4)$ \\
\hline $\mathrm{O}(1)-\mathrm{U}(1)-\mathrm{F}(2) \# 2$ & $81.6(2)$ & $\mathrm{P}(1)-\mathrm{O}(2)-\mathrm{U}(1)$ & $139.6(4)$ \\
\hline
\end{tabular}




\begin{tabular}{|c|c|c|c|}
\hline $\mathrm{O}(2)-\mathrm{U}(1)-\mathrm{F}(2) \# 2$ & $72.6(2)$ & $\mathrm{U}(1)-\mathrm{F}(2)-\mathrm{U}(1) \# 2$ & $1113.38(19$ \\
\hline $\mathrm{F}(2)-\mathrm{U}(1)-\mathrm{F}(2) \# 2$ & $66.62(19)$ & $\mathrm{P}(1) \# 1-\mathrm{O}(3)-\mathrm{U}(1)$ & $142.5(4)$ \\
\hline $\mathrm{O}(3)-\mathrm{U}(1)-\mathrm{F}(2) \# 2$ & $141.2(2)$ & $\mathrm{U}(1)-\mathrm{F}(3)-\mathrm{U}(1) \# 1$ & $114.8(2)$ \\
\hline $\mathrm{F}(3)-\mathrm{U}(1)-\mathrm{F}(2) \# 2$ & $143.24(17)$ & & \\
\hline \multicolumn{4}{|c|}{ Compound 2} \\
\hline $\mathrm{F}(1)-\mathrm{U}(1)-\mathrm{O}(1)$ & $79.6(2)$ & $\mathrm{F}(3) \# 1-\mathrm{U}(1)-\mathrm{F}(2) \# 2$ & $140.26(15)$ \\
\hline $\mathrm{F}(1)-\mathrm{U}(1)-\mathrm{F}(2)$ & $77.58(18)$ & $\mathrm{F}(1)-\mathrm{U}(1)-\mathrm{U}(1) \# 2$ & $73.12(15)$ \\
\hline $\mathrm{O}(1)-\mathrm{U}(1)-\mathrm{F}(2)$ & $141.1(2)$ & $\mathrm{O}(1)-\mathrm{U}(1)-\mathrm{U}(1) \# 2$ & $108.81(16)$ \\
\hline $\mathrm{F}(1)-\mathrm{U}(1)-\mathrm{O}(3)$ & $71.8(2)$ & $\mathrm{F}(2)-\mathrm{U}(1)-\mathrm{U}(1) \# 2$ & $33.93(11)$ \\
\hline $\mathrm{O}(1)-\mathrm{U}(1)-\mathrm{O}(3)$ & $106.0(2)$ & $\mathrm{O}(3)-\mathrm{U}(1)-\mathrm{U}(1) \# 2$ & $124.10(15)$ \\
\hline $\mathrm{F}(2)-\mathrm{U}(1)-\mathrm{O}(3)$ & $96.27(18)$ & $\mathrm{F}(3)-\mathrm{U}(1)-\mathrm{U}(1) \# 2$ & $102.54(11)$ \\
\hline $\mathrm{F}(1)-\mathrm{U}(1)-\mathrm{F}(3)$ & $133.89(17)$ & $\mathrm{O}(2)-\mathrm{U}(1)-\mathrm{U}(1) \# 2$ & $84.67(16)$ \\
\hline $\mathrm{O}(1)-\mathrm{U}(1)-\mathrm{F}(3)$ & $140.22(19)$ & $\mathrm{F}(3) \# 1-\mathrm{U}(1)-\mathrm{U}(1) \# 2$ & $156.75(11)$ \\
\hline$F(2)-U(1)-F(3)$ & $76.16(16)$ & $\mathrm{F}(2) \# 2-\mathrm{U}(1)-\mathrm{U}(1) \# 2$ & $31.35(10)$ \\
\hline $\mathrm{O}(3)-\mathrm{U}(1)-\mathrm{F}(3)$ & $74.21(18)$ & $\mathrm{F}(1)-\mathrm{U}(1)-\mathrm{U}(1) \# 1$ & $142.28(14)$ \\
\hline $\mathrm{F}(1)-\mathrm{U}(1)-\mathrm{O}(2)$ & $144.73(19)$ & $\mathrm{O}(1)-\mathrm{U}(1)-\mathrm{U}(1) \# 1$ & $108.06(15)$ \\
\hline $\mathrm{O}(1)-\mathrm{U}(1)-\mathrm{O}(2)$ & $82.2(2)$ & $\mathrm{F}(2)-\mathrm{U}(1)-\mathrm{U}(1) \# 1$ & $109.30(11)$ \\
\hline $\mathrm{F}(2)-\mathrm{U}(1)-\mathrm{O}(2)$ & $99.0(2)$ & $\mathrm{O}(3)-\mathrm{U}(1)-\mathrm{U}(1) \# 1$ & $70.59(14)$ \\
\hline $\mathrm{O}(3)-\mathrm{U}(1)-\mathrm{O}(2)$ & $142.8(2)$ & $\mathrm{F}(3)-\mathrm{U}(1)-\mathrm{U}(1) \# 1$ & $33.14(11)$ \\
\hline $\mathrm{F}(3)-\mathrm{U}(1)-\mathrm{O}(2)$ & $76.89(18)$ & $\mathrm{O}(2)-\mathrm{U}(1)-\mathrm{U}(1) \# 1$ & $72.38(14)$ \\
\hline $\mathrm{F}(1)-\mathrm{U}(1)-\mathrm{F}(3) \# 1$ & $129.91(18)$ & $\mathrm{F}(3) \# 1-\mathrm{U}(1)-\mathrm{U}(1) \# 1$ & $31.89(10)$ \\
\hline $\mathrm{O}(1)-\mathrm{U}(1)-\mathrm{F}(3) \# 1$ & $76.79(18)$ & $\mathrm{F}(2) \# 2-\mathrm{U}(1)-\mathrm{U}(1) \# 1$ & $143.10(10)$ \\
\hline $\mathrm{F}(2)-\mathrm{U}(1)-\mathrm{F}(3) \# 1$ & $141.19(15)$ & $\mathrm{U}(1) \# 2-\mathrm{U}(1)-\mathrm{U}(1) \# 1$ & $132.89(2)$ \\
\hline $\mathrm{O}(3)-\mathrm{U}(1)-\mathrm{F}(3) \# 1$ & $73.26(18)$ & $\mathrm{O}(3)-\mathrm{P}(1)-\mathrm{O}(2) \# 1$ & 112.1(3) \\
\hline $\mathrm{F}(3)-\mathrm{U}(1)-\mathrm{F}(3) \# 1$ & $65.04(18)$ & $\mathrm{O}(3)-\mathrm{P}(1)-\mathrm{O}(1) \# 3$ & $111.2(3)$ \\
\hline $\mathrm{O}(2)-\mathrm{U}(1)-\mathrm{F}(3) \# 1$ & $73.58(19)$ & $\mathrm{O}(2) \# 1-\mathrm{P}(1)-\mathrm{O}(1) \# 3$ & $110.0(3)$ \\
\hline $\mathrm{F}(1)-\mathrm{U}(1)-\mathrm{F}(2) \# 2$ & $74.21(18)$ & $\mathrm{P}(1) \# 4-\mathrm{O}(1)-\mathrm{U}(1)$ & $163.0(4)$ \\
\hline $\mathrm{O}(1)-\mathrm{U}(1)-\mathrm{F}(2) \# 2$ & 78.41(19) & $\mathrm{U}(1)-\mathrm{F}(2)-\mathrm{U}(1) \# 2$ & $114.72(17)$ \\
\hline
\end{tabular}




\begin{tabular}{|c|c|c|c|}
\hline $\mathrm{F}(2)-\mathrm{U}(1)-\mathrm{F}(2) \# 2$ & $65.28(17)$ & $\mathrm{P}(1) \# 1-\mathrm{O}(2)-\mathrm{U}(1)$ & $134.2(4)$ \\
\hline $\mathrm{O}(3)-\mathrm{U}(1)-\mathrm{F}(2) \# 2$ & $144.16(17)$ & $\mathrm{P}(1)-\mathrm{O}(3)-\mathrm{U}(1)$ & $135.5(3)$ \\
\hline $\mathrm{F}(3)-\mathrm{U}(1)-\mathrm{F}(2) \# 2$ & $125.20(16)$ & $\mathrm{U}(1)-\mathrm{F}(3)-\mathrm{U}(1) \# 1$ & $114.96(18)$ \\
\hline $\mathrm{O}(2)-\mathrm{U}(1)-\mathrm{F}(2) \# 2$ & $72.68(18)$ & & \\
\hline \multicolumn{4}{|c|}{ Compound 3} \\
\hline $\mathrm{O}(1)-\mathrm{U}(1)-\mathrm{O}(2)$ & $176.3(4)$ & $\mathrm{O}(4) \# 1-\mathrm{U}(1)-\mathrm{F}(1)$ & $151.0(3)$ \\
\hline $\mathrm{O}(1)-\mathrm{U}(1)-\mathrm{O}(3)$ & $91.5(4)$ & $\mathrm{O}(5) \# 2-\mathrm{U}(1)-\mathrm{F}(1)$ & $131.3(2)$ \\
\hline $\mathrm{O}(2)-\mathrm{U}(1)-\mathrm{O}(3)$ & $90.6(4)$ & $\mathrm{F}(1) \# 3-\mathrm{U}(1)-\mathrm{F}(1)$ & $63.6(3)$ \\
\hline $\mathrm{O}(1)-\mathrm{U}(1)-\mathrm{O}(4) \# 1$ & $94.2(4)$ & $\mathrm{O}(1)-\mathrm{U}(1)-\mathrm{U}(1) \# 3$ & $90.9(3)$ \\
\hline $\mathrm{O}(2)-\mathrm{U}(1)-\mathrm{O}(4) \# 1$ & $89.3(4)$ & $\mathrm{O}(2)-\mathrm{U}(1)-\mathrm{U}(1) \# 3$ & $85.6(3)$ \\
\hline $\mathrm{O}(3)-\mathrm{U}(1)-\mathrm{O}(4) \# 1$ & $80.0(3)$ & $\mathrm{O}(3)-\mathrm{U}(1)-\mathrm{U}(1) \# 3$ & $103.3(2)$ \\
\hline $\mathrm{O}(1)-\mathrm{U}(1)-\mathrm{O}(5) \# 2$ & $89.5(4)$ & $\mathrm{O}(4) \# 1-\mathrm{U}(1)-\mathrm{U}(1) \# 3$ & $173.9(2)$ \\
\hline $\mathrm{O}(2)-\mathrm{U}(1)-\mathrm{O}(5) \# 2$ & $89.7(4)$ & $\mathrm{O}(5) \# 2-\mathrm{U}(1)-\mathrm{U}(1) \# 3$ & $99.71(19)$ \\
\hline $\mathrm{O}(3)-\mathrm{U}(1)-\mathrm{O}(5) \# 2$ & $156.9(3)$ & $\mathrm{F}(1) \# 3-\mathrm{U}(1)-\mathrm{U}(1) \# 3$ & $31.95(15)$ \\
\hline $\mathrm{O}(4) \# 1-\mathrm{U}(1)-\mathrm{O}(5) \# 2$ & $76.9(3)$ & $\mathrm{F}(1)-\mathrm{U}(1)-\mathrm{U}(1) \# 3$ & $31.70(15)$ \\
\hline $\mathrm{O}(1)-\mathrm{U}(1)-\mathrm{F}(1) \# 3$ & $88.9(4)$ & $\mathrm{O}(3)-\mathrm{P}(1)-\mathrm{O}(4)$ & $113.2(5)$ \\
\hline $\mathrm{O}(2)-\mathrm{U}(1)-\mathrm{F}(1) \# 3$ & $87.4(3)$ & $\mathrm{O}(3)-\mathrm{P}(1)-\mathrm{O}(5)$ & $107.1(5)$ \\
\hline $\mathrm{O}(3)-\mathrm{U}(1)-\mathrm{F}(1) \# 3$ & $135.3(3)$ & $\mathrm{O}(4)-\mathrm{P}(1)-\mathrm{O}(5)$ & $111.8(5)$ \\
\hline $\mathrm{O}(4) \# 1-\mathrm{U}(1)-\mathrm{F}(1) \# 3$ & $144.6(3)$ & $\mathrm{P}(1)-\mathrm{O}(3)-\mathrm{U}(1)$ & $166.0(6)$ \\
\hline $\mathrm{O}(5) \# 2-\mathrm{U}(1)-\mathrm{F}(1) \# 3$ & $67.8(2)$ & $\mathrm{P}(1)-\mathrm{O}(4)-\mathrm{U}(1) \# 1$ & $136.6(5)$ \\
\hline $\mathrm{O}(1)-\mathrm{U}(1)-\mathrm{F}(1)$ & $92.6(4)$ & $\mathrm{P}(1)-\mathrm{O}(5)-\mathrm{U}(1) \# 4$ & $136.6(4)$ \\
\hline $\mathrm{O}(2)-\mathrm{U}(1)-\mathrm{F}(1)$ & $85.1(3)$ & $\mathrm{U}(1) \# 3-\mathrm{F}(1)-\mathrm{U}(1)$ & $116.4(3)$ \\
\hline $\mathrm{O}(3)-\mathrm{U}(1)-\mathrm{F}(1)$ & $71.7(3)$ & & \\
\hline
\end{tabular}

\footnotetext{
Symmetry transformations used to generate equivalent atoms for 1 : \#1 -x+1,-y+2,-z+1 \#2-x+1,-y+1,-z+1 $\# 3-x+2,-y+2,-z+1 \quad \# 4-x,-y+1,-z+2$; for $2: \# 1-x+1,-y+2,-z+2 \quad \# 2-x+2,-y+2,-z+2 \quad \# 3-x+1, y-1 / 2,-z+3 / 2 \quad \# 4$ $-\mathrm{x}+1, \mathrm{y}+1 / 2,-\mathrm{z}+3 / 2 \quad \# 5-\mathrm{x},-\mathrm{y}+2,-\mathrm{z}+1$; for $3: \# 1-\mathrm{x}+1,-\mathrm{y}+1,-\mathrm{z}+1 \quad \# 2 \mathrm{x}, \mathrm{y}-1, \mathrm{z} \quad \# 3-\mathrm{x}+2,-\mathrm{y}+1,-\mathrm{z}+2 \quad \# 4 \mathrm{x}, \mathrm{y}+1, \mathrm{z}$ $\# 5-x+1,-y+2,-z+2$.
} 


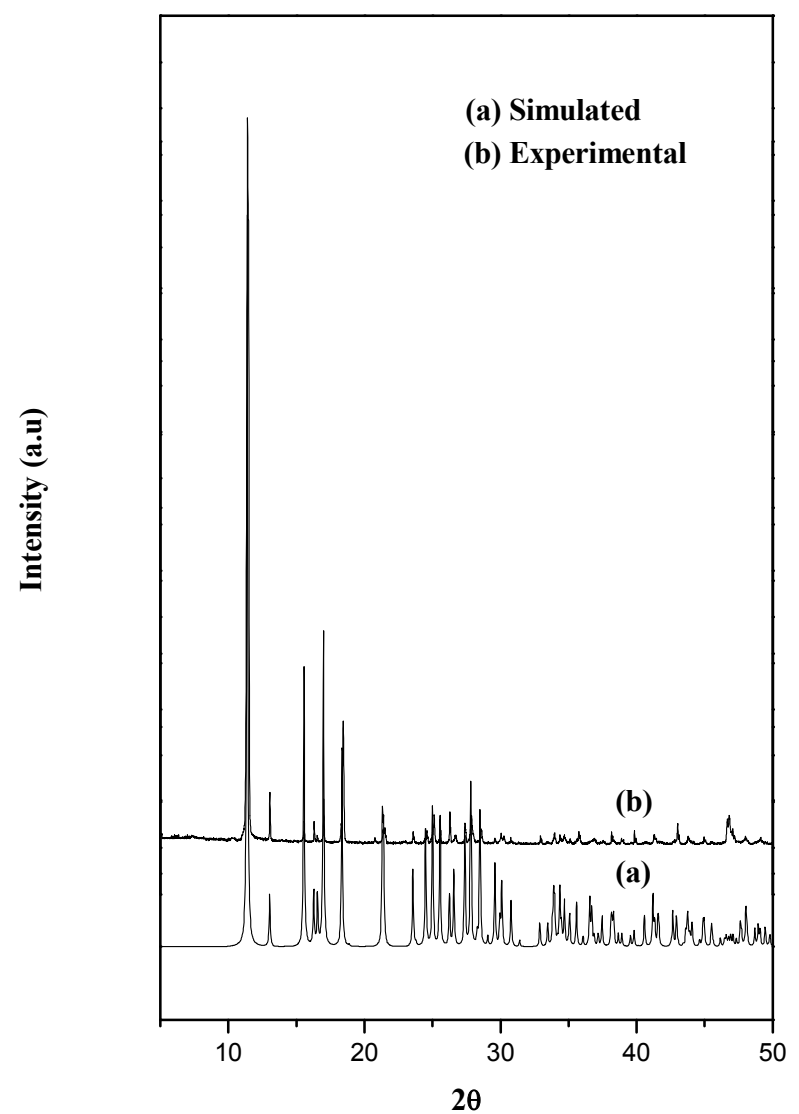

Fig S1: Simulated and experimental XRD pattern for $\left[\mathrm{C}_{2} \mathrm{~N}_{2} \mathrm{H}_{10}\right]\left[\mathrm{U}_{2}{ }^{\mathrm{IV}} \mathrm{F}_{6}\left(\mathrm{HPO}_{3}\right)_{2}\right], \mathbf{1}$. 


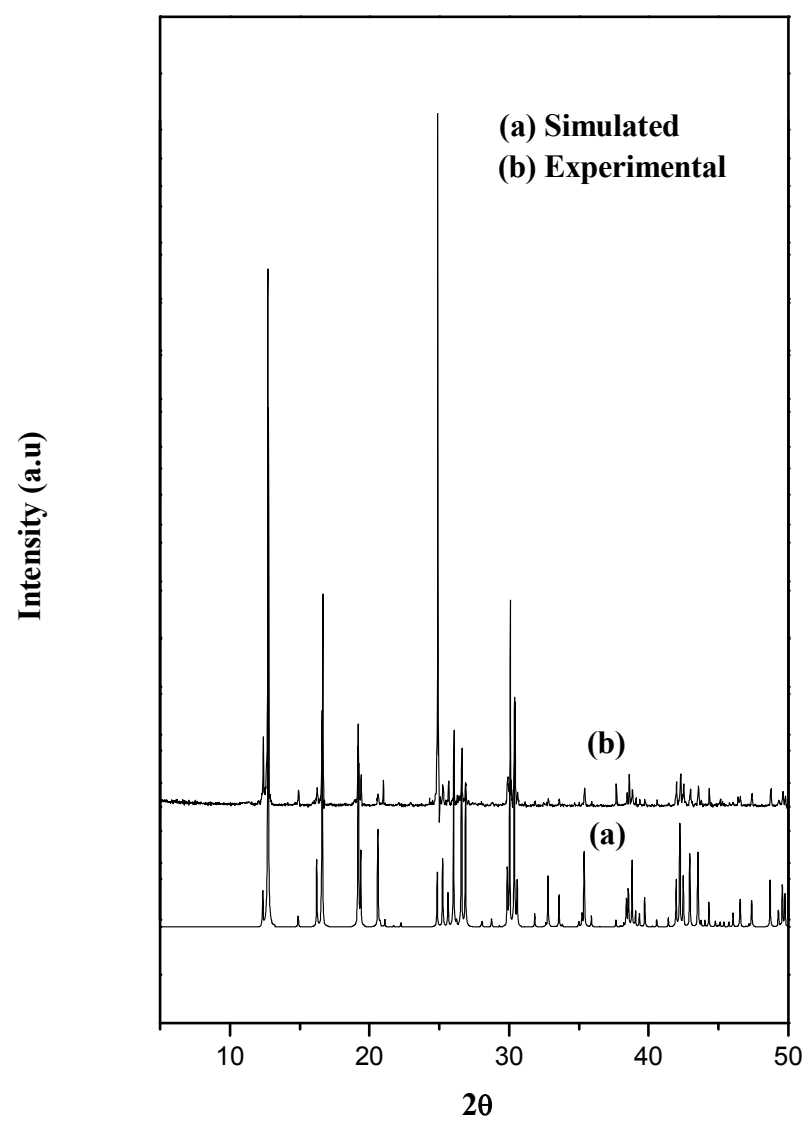

Fig S2: Simulated and experimental XRD pattern for $\left[\mathrm{C}_{4} \mathrm{~N}_{2} \mathrm{H}_{12}\right]\left[\mathrm{U}_{2}{ }^{\mathrm{IV}} \mathrm{F}_{6}\left(\mathrm{HPO}_{3}\right)_{2}\right], \mathbf{2}$. 


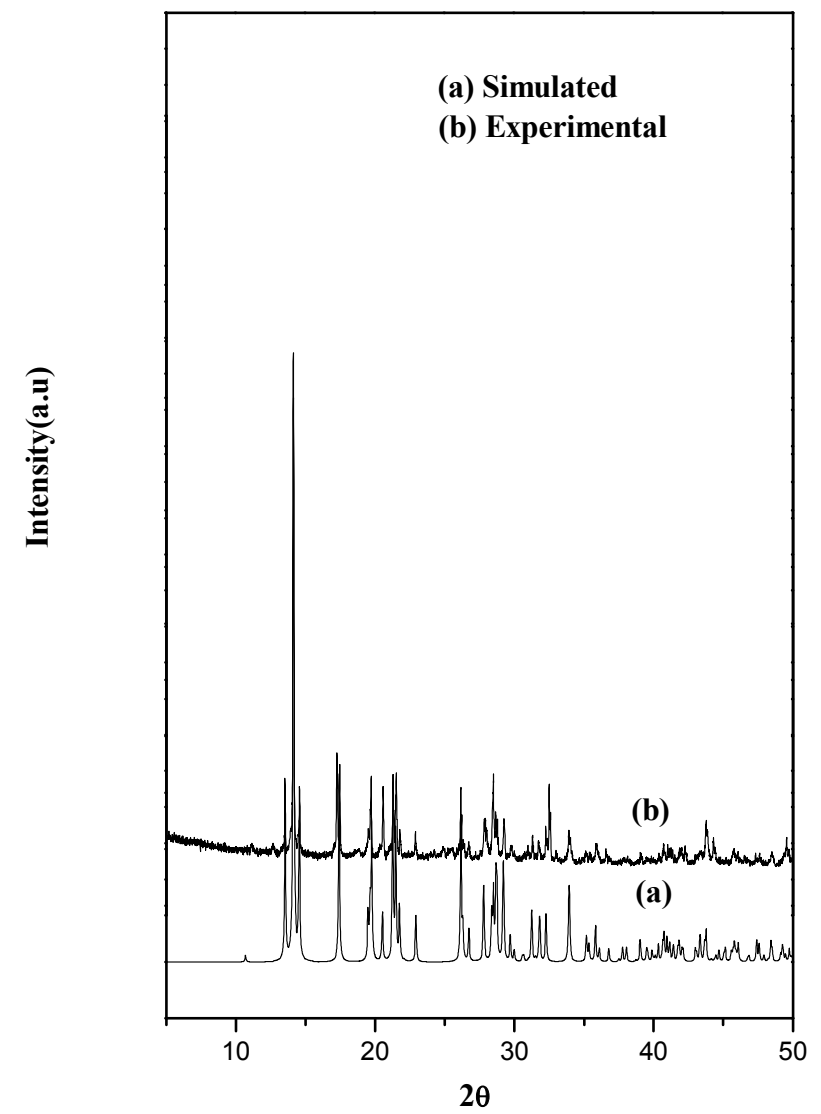

Fig S3: Simulated and experimental XRD pattern for $\left[\mathrm{C}_{4} \mathrm{~N}_{2} \mathrm{H}_{12}\right]\left[\left(\mathrm{U}^{\mathrm{VI}} \mathrm{O}_{2}\right)_{2} \mathrm{~F}_{2}\left(\mathrm{HPO}_{3}\right)_{2}\right], 3$. 


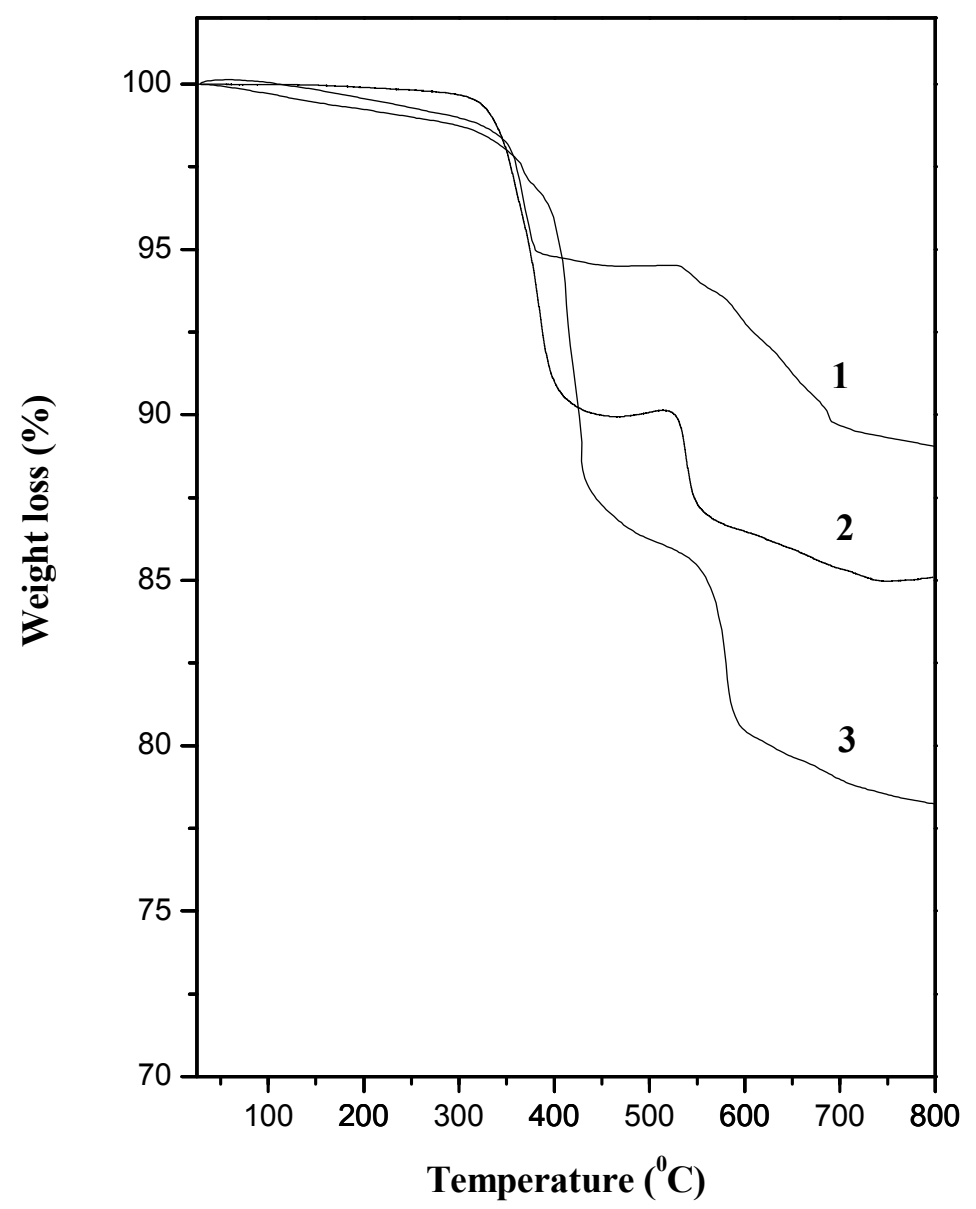

Fig S4: Thermogravimetric analysis curve for the compounds, $\left[\mathrm{C}_{2} \mathrm{~N}_{2} \mathrm{H}_{10}\right]\left[\mathrm{U}_{2}{ }^{\mathrm{IV}} \mathrm{F}_{6}\left(\mathrm{HPO}_{3}\right)_{2}\right], \mathbf{1}$, $\left[\mathrm{C}_{4} \mathrm{~N}_{2} \mathrm{H}_{12}\right]\left[\mathrm{U}_{2}{ }^{\mathrm{IV}} \mathrm{F}_{6}\left(\mathrm{HPO}_{3}\right)_{2}\right], 2$ and $\left[\mathrm{C}_{4} \mathrm{~N}_{2} \mathrm{H}_{12}\right]\left[\left(\mathrm{U}^{\mathrm{VI}} \mathrm{O}_{2}\right)_{2} \mathrm{~F}_{2}\left(\mathrm{HPO}_{3}\right)_{2}\right], \mathbf{3}$.. 


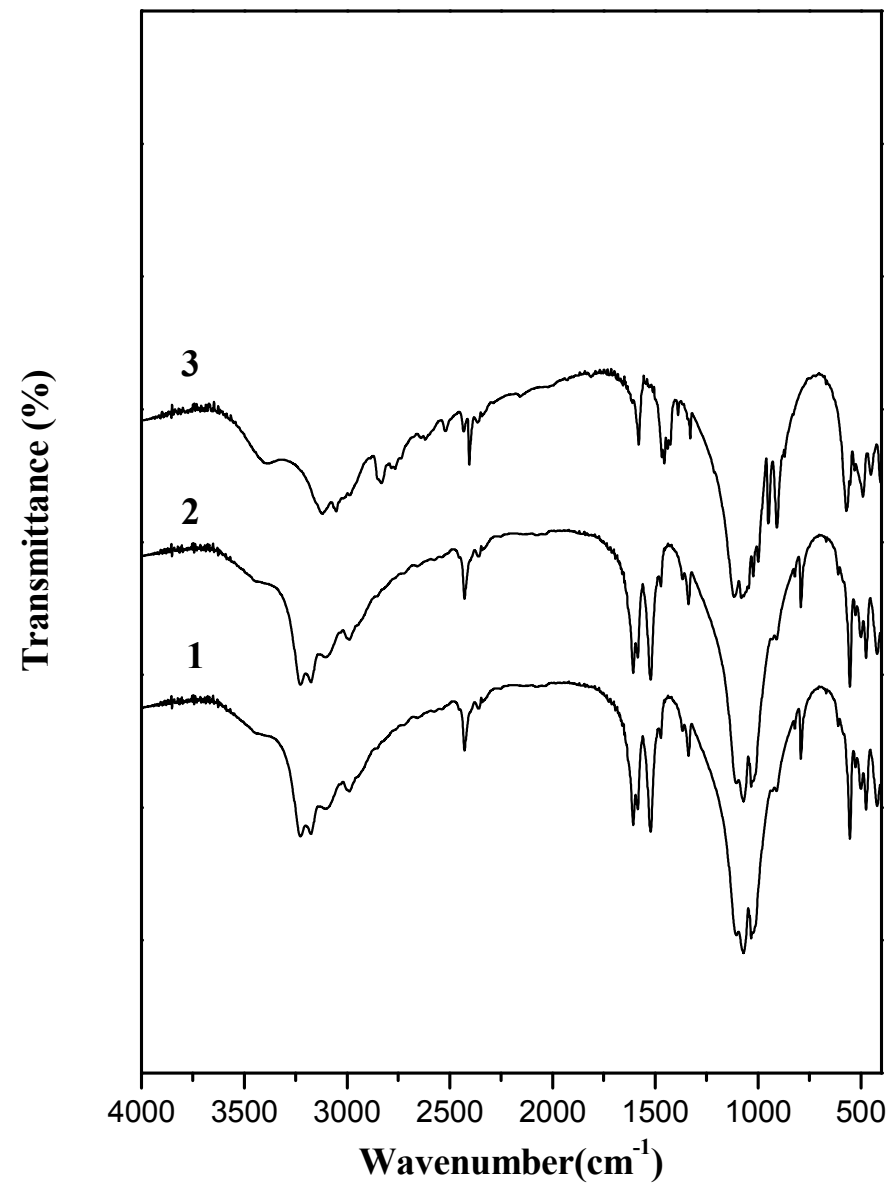

Fig S5: Infrared spectra of the compounds $\left[\mathrm{C}_{2} \mathrm{~N}_{2} \mathrm{H}_{10}\right]\left[\mathrm{U}_{2}^{\mathrm{IV}} \mathrm{F}_{6}\left(\mathrm{HPO}_{3}\right)_{2}\right], \mathbf{1},\left[\mathrm{C}_{4} \mathrm{~N}_{2} \mathrm{H}_{12}\right]\left[\mathrm{U}_{2}{ }^{\mathrm{IV}} \mathrm{F}_{6}\left(\mathrm{HPO}_{3}\right)_{2}\right], \mathbf{2}$ and $\left[\mathrm{C}_{4} \mathrm{~N}_{2} \mathrm{H}_{12}\right]\left[\left(\mathrm{U}^{\mathrm{VI}} \mathrm{O}_{2}\right)_{2} \mathrm{~F}_{2}\left(\mathrm{HPO}_{3}\right)_{2}\right], \mathbf{3}$.. 
(a)

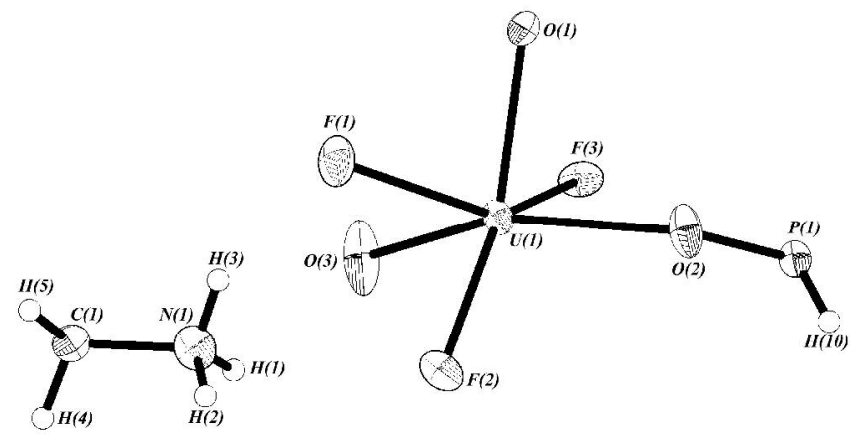

(b)
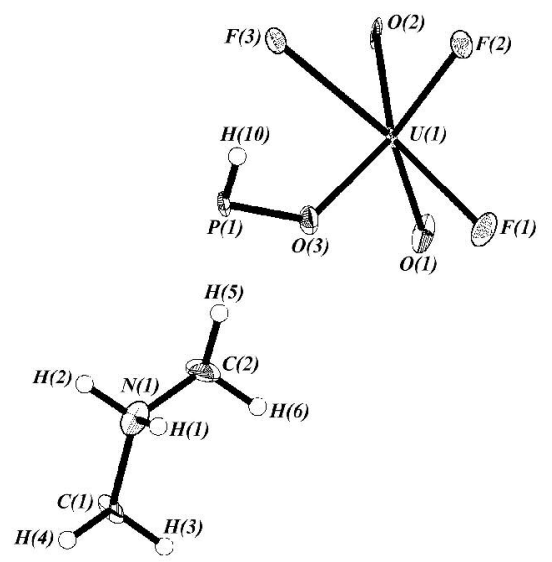

(c)
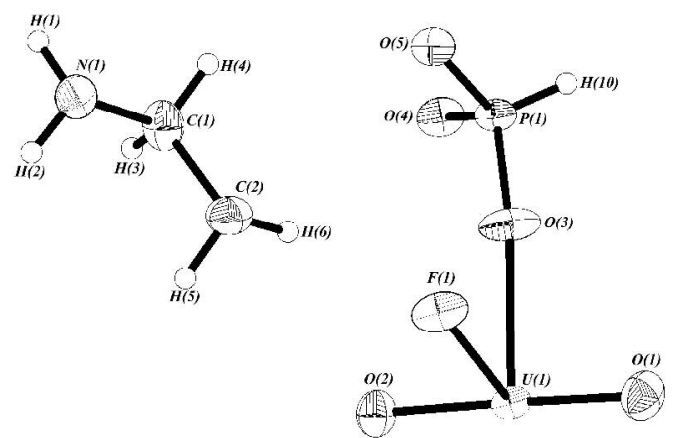

Fig. S6: ORTEP diagram of (a) 1, (b) 2 and (c) 3. Thermal ellipsoids are given at $50 \%$ probability. 
(a)

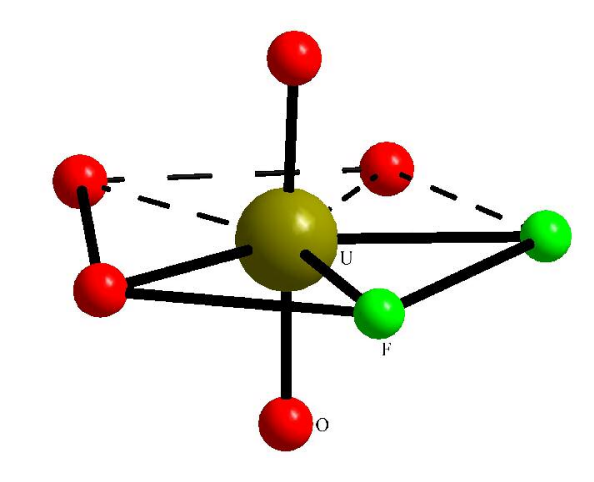

(b)

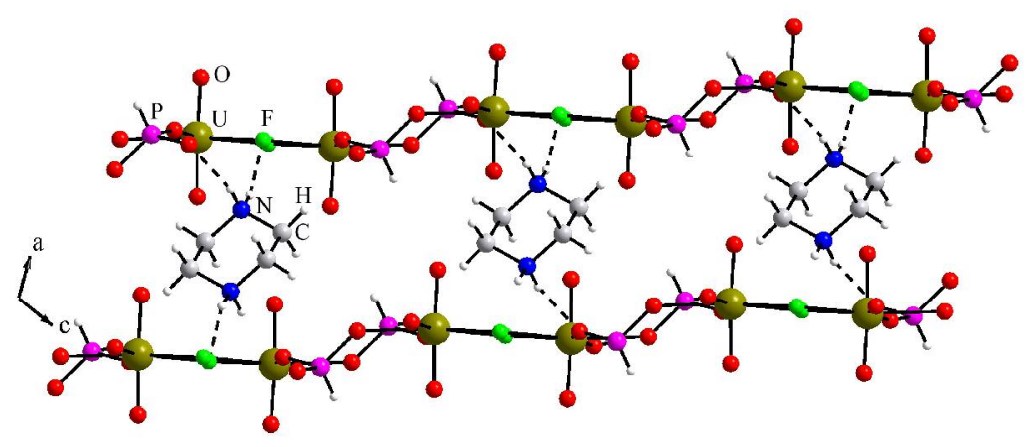

Fig. S7 (a) Coordination geometry around $\mathrm{U}^{6+}$ ions in $\left[\mathrm{C}_{4} \mathrm{~N}_{2} \mathrm{H}_{12}\right]\left[\left(\mathrm{U}^{\mathrm{VI}} \mathrm{O}_{2}\right)_{2} \mathrm{~F}_{2}\left(\mathrm{HPO}_{3}\right)_{2}\right], \mathbf{3}$.

(b) The structure of $\mathbf{3}$ viewed along ac plane, showing the arrangement of the layers and the piperazine molecules. The dotted lines represent the possible hydrogen bond interactions. 